OPEN ACCESS

Edited by:

Ahmed Rebai,

Centre of Biotechnology of Sfax,

Tunisia

Reviewed by:

James A. Poulter,

University of Leeds, United Kingdom

Nancy Monroy-Jaramillo,

National Institute of Neurology

and Neurosurgery, Mexico

*Correspondence:

Bin Wang

gzwangbin@smu.edu.cn

Victor Wei Zhang

victor.w.zhang@amcarelab.com.cn

Specialty section:

This article was submitted to Human and Medical Genomics,

a section of the journal

Frontiers in Genetics

Received: 15 June 2021

Accepted: 30 July 2021

Published: 19 August 2021

Citation:

Ouyang $X$, Zhang $Y$, Zhang $L$, Luo J, Zhang T, Hu H, Liu L, Zhong L, Zeng S, Xu P, Bai Z, Wong L-J,

Wang J, Wang $C$, Wang $B$ and

Zhang VW (2021) Clinical Utility

of Rapid Exome Sequencing Combined With Mitochondrial DNA Sequencing in Critically III Pediatric

Patients With Suspected Genetic Disorders. Front. Genet. 12:725259. doi: 10.3389/fgene.2021.725259

\section{Clinical Utility of Rapid Exome Sequencing Combined With Mitochondrial DNA Sequencing in Critically III Pediatric Patients With Suspected Genetic Disorders}

Xuejun Ouyang', Yu Zhang', Lijuan Zhang ${ }^{1}$, Jixuan Luo', Ting Zhang' ${ }^{2}$, Hui Hu², Lin Liü ${ }^{3}$, Lieqiang Zhong ${ }^{3}$, Shaoying Zeng ${ }^{3}$, Pingyi $\mathrm{Xu}^{4}$, Zhenjiang Bai ${ }^{5}$, Lee-Jun Wong ${ }^{6}$, Jing Wang ${ }^{6,7}$, Chunli Wang ${ }^{7}$, Bin Wang ${ }^{1 *}$ and Victor Wei Zhang ${ }^{6,7 *}$

'Department of Pediatrics, Zhujiang Hospital, Southern Medical University, Guangzhou, China, ${ }^{2}$ Department of Gastroenterology, Shanghai Children's Hospital, Shanghai, China, ${ }^{3}$ Department of Vasculocardiology, Guangdong Provincial People's Hospital, Guangzhou, China, ${ }^{4}$ Department of Neurology, First Affiliated Hospital of Guangzhou Medical University, Guangzhou, China, ${ }^{5}$ Department of Critical Care Medicine, Children's Hospital of Soochow University, Suzhou, China, ${ }^{6}$ Department of Human and Molecular Genetics, Baylor College of Medicine, Houston, TX, United States, ${ }^{7}$ AmCare Genomics Lab, Guangzhou, China

Genetic disorders are a frequent cause of hospitalization, morbidity and mortality in pediatric patients, especially in the neonatal or pediatric intensive care unit (NICU/PICU). In recent years, rapid genome-wide sequencing (exome or whole genome sequencing) has been applied in the NICU/PICU. However, mtDNA sequencing is not routinely available in rapid genetic diagnosis programs, which may fail to diagnose mtDNA mutation-associated diseases. Herein, we explored the clinical utility of rapid exome sequencing combined with mtDNA sequencing in critically ill pediatric patients with suspected genetic disorders. Rapid clinical exome sequencing (CES) was performed as a first-tier test in 40 critically ill pediatric patients (aged from 6 days to 15 years) with suspected genetic conditions. Blood samples were also collected from the parents for trio analysis. Twenty-six patients presented with neuromuscular abnormalities or other systemic abnormalities, suggestive of suspected mitochondrial diseases or the necessity for a differential diagnosis of other diseases, underwent rapid mtDNA sequencing concurrently. A diagnosis was made in 18 patients (45.0\%, 18/40); three cases with de novo autosomal dominant variants, ten cases with homozygous or compound heterozygous variants, three cases with hemizygous variants inherited from mother, three cases with heterozygous variants inherited from either parent, and one case with a mtDNA mutation. The 18 patients were diagnosed with metabolic $(n=7)$, immunodeficiency $(n=4)$, cardiovascular $(n=2)$, neuromuscular $(n=2)$ disorders, and others. Genetic testing reports were generated with a median time of 5 days (range, 39 days). Thirteen patients that were diagnosed had an available medical treatment and 
resulted in a positive outcome. We propose that rapid exome sequencing combined with mitochondrial DNA sequencing should be available to patients with suspected mitochondrial diseases or undefined clinical features necessary for making a differential diagnosis of other diseases.

Keywords: rapid exome sequencing, mitochondrial diseases, mtDNA sequencing, pediatric patients, genetic disorders

\section{INTRODUCTION}

Genetic disorders are the leading cause of hospitalization, morbidity and mortality in pediatric patients, especially in the neonatal or pediatric intensive care unit (NICU/PICU) (Stevenson and Carey, 2004; Willig et al., 2015; French et al., 2019). A genetic diagnosis in the critical illness remains a great challenge for the physicians when the patients' phenotypes has not fully been expressed due to age to make a clear clinical diagnosis. Moreover, many traditional diagnostic methods are too slow to provide clinically useful information to physicians. However, an early and accurate genetic diagnosis would be of great value for the clinical management of the patient and family (Australian Genomics Health Alliance Acute Care Flagship, Lunke et al., 2020; Cakici et al., 2020; Dimmock et al., 2020). For those genetic diseases that can be treated, rapid diagnosis can provide timely interventions and avoid other unnecessary or potentially harmful therapies, thus reducing mortality and intensive care. For genetic diseases currently lacking effective treatments, the diagnosis may be useful in evaluating prognosis and preventing possible complications, as well as facilitating further genetic counseling for the family.

The recent development of rapid genome-wide sequencing (exome or whole genome sequencing, ES or WGS) has been widely used for genetic analyses in critically ill pediatric patients with suspected genetic disorders in the PICU/NICU (Saunders et al., 2012; Petrikin et al., 2015, 2018; Kingsmore et al., 2019). Generally, rapid exome sequencing (WES or gene panels) has delivered a molecular diagnosis for patients with the turnaround time (TAT) of 1-2 weeks, and positively changed the medical management (Elliott et al., 2019; Gubbels et al., 2020; Smigiel et al., 2020). The molecular diagnosis applied by rapid WGS was generally completed less than one week, even within $24 \mathrm{~h}$, potentially revolutionizing clinical practice (Clark et al., 2019; Wang et al., 2020). ES focuses on targeted sequencing of the protein-coding regions of the nuclear genome, while WGS involves sequencing the entire genome including coding regions, intergenic regions and the mitochondrial genome. Previous studies reported that most of the patients identified as genetic conditions by rapid ES or WGS were monogenic disorders, and just a few cases who underwent WGS were diagnosed with a mitochondrial disorder caused by a single nucleotide variant (SNV) in mitochondrial DNA (mtDNA) (Petrikin et al., 2015; Willig et al., 2015).

Mitochondrial disorders are a group of complicated and heterogeneous diseases caused by mutations in genes that lead to defective oxidative phosphorylation (OXPHOS) and ATP synthesis (Wortmann et al., 2017). The diagnosis of mitochondrial disorders has always been challenging due to the clinical features being variable. Although the central nervous system (CNS) is most often involved, other single organ systems or multiple organ systems may be affected, such as muscle, liver, heart, kidneys, gastrointestinal tract, endocrine glands, and others (Kisler et al., 2010; Schon et al., 2020). Therefore, mitochondrial disorders enter the differential diagnosis of many common diseases. Mitochondrial diseases with childhood onset often present in the newborn period, however, it is very difficult to make an early diagnosis because the clinical phenotypes overlap with other common disorders (Tulinius and Oldfors, 2011; Honzik et al., 2012). The multimeric protein complexes comprising the OXPHOS system have a dual genetic origin (nuclear DNA or mtDNA), while the majority of subunits are encoded by the nuclear genome that can be identified effectively by using ES or WGS. However, mitochondrial genome sequencing is not routinely available in rapid genetic diagnosis programs, which may fail to diagnose mtDNA mutation-associated diseases.

In this study, we explored the clinical utility of combined rapid clinical exome sequencing (CES) and mtDNA sequencing in 40 critically ill pediatric patients with suspected genetic disorders. The CES in this study was a uniquely designed subexome panel comprised of approximately 5000 known genes causing Mendelian diseases, while whole mtDNA sequencing was performed using long-PCR followed by next generation sequencing (NGS).

\section{METHODS}

\section{Patient Cohort}

This multi-center study was performed at five hospitals in China from June 2018 to May 2020, which was approved by the Ethical Review Committee of the hospitals. The enrollment criteria were as follows: (1) pediatric patients with a severe and/or progressive disease but without a definite diagnosis, such as metabolic disturbances, neurological abnormalities, immune system defects, respiratory and/or cardiovascular failure and multiple congenital anomalies; (2) written informed consent was obtained from both parents prior to genetic testing; (3) both parents were available, and rapid CES was applied as a first-tier genetic test for a trio analysis. A total of 40 patients were enrolled in this study, including 29 patients in PICU/NICU and 11 patients in the other pediatric wards (Table 1). Twenty-six patients with neuromuscular abnormalities or other systemic abnormalities underwent rapid mtDNA 
TABLE 1 | General description of the 40 pediatric patients with rapid genetic diagnosis.

\begin{tabular}{|c|c|c|}
\hline Characteristic & Total cases $(n=40)$ & Diagnosed by genetic analysis $(n=18)$ \\
\hline \multicolumn{3}{|l|}{ Sex } \\
\hline Male & $24(60.0 \%, 24 / 40)$ & $11(61.1 \%, 11 / 18)$ \\
\hline Female & $16(40.0 \%, 16 / 40)$ & $7(38.9 \%, 7 / 18)$ \\
\hline \multicolumn{3}{|l|}{ Age at genetic testing ( $1 \mathrm{~d} \sim 15 \mathrm{y})$} \\
\hline Neonates: Age $<28$ d & $6(15.0 \%, 6 / 40)$ & $4(22.2 \%, 4 / 18)$ \\
\hline Infants: $28 \mathrm{~d} \leq$ Age $<1$ y & $25(62.5 \%, 25 / 40)$ & $12(66.7 \%, 12 / 18)$ \\
\hline Children: Age $\geq 1$ y & $9(22.5 \%, 9 / 40)$ & $2(11.1 \%, 2 / 18)$ \\
\hline \multicolumn{3}{|l|}{ Patients from departments } \\
\hline Neonatal intensive care unit (NICU) & $18(45.0 \%, 18 / 40)$ & $7(36.8 \%, 7 / 18)$ \\
\hline Pediatric intensive care unit (PICU) & $11(27.5 \%, 11 / 40)$ & $7(36.8 \%, 7 / 18)$ \\
\hline Other pediatric wards & $11(27.5 \%, 11 / 40)$ & $4(21.1 \%, 4 / 18)$ \\
\hline \multicolumn{3}{|c|}{ Classification based on clinical phenotypes at genetic testing } \\
\hline Neuromuscular diseases & $5(12.5 \%, 5 / 40)$ & $4(22.2 \%, 4 / 18)$ \\
\hline Digestive diseases & $4(10.0 \%, 4 / 40)$ & 0 \\
\hline Cardiovascular diseases & $4(10.0 \%, 4 / 40)$ & $2(11.1 \%, 2 / 18)$ \\
\hline Endocrine diseases & $3(7.5 \%, 3 / 40)$ & $2(11.1 \%, 2 / 18)$ \\
\hline Metabolic-related diseases & $3(7.5 \%, 3 / 40)$ & $3(16.7 \%, 3 / 18)$ \\
\hline Immunodeficiency diseases & $2(5.0 \%, 2 / 40)$ & $1(5.6 \%, 1 / 18)$ \\
\hline Multi-system diseases & $19(47.5 \%, 19 / 40)$ & $6(33.3 \%, 6 / 18)$ \\
\hline
\end{tabular}

CES, clinical exome sequencing; mtDNA, mitochondrial DNA; d, day; y, year.

sequencing concurrently. The flowchart of this study was shown in Figure 1.

\section{Clinical Exome Sequencing}

Peripheral blood samples of the patients and their parents were collected and the genomic DNA was extracted using the SolPure Blood DNA kit (Magen) according to the manufacturer's instructions. The Q800R Sonicator (Qsonica) was used to disrupt the genomic DNA to generate $300-500$ bp fragments. The paired-end libraries were prepared following the Illumina library preparation protocol. Custom-designed NimbleGen SeqCap probes (Roche NimbleGen, Madison, WI) were used for insolution hybridization to enrich target sequences, which included $\sim 5000$ genes associated with known Mendelian genetic diseases based on databases of Online Mendelian Inheritance in Man (OMIM), Human Gene Mutation Database (HGMD), and literature (AmCare Genomic Laboratory, Guangzhou, China). The NextSeq500 or NovaSeq 6000 sequencer (Illumina, San Diego, CA) was used for sequencing of enriched DNA. The average coverage depth was $200 \times$, and at least $98 \%$ of the target regions were covered by $20 \times$. The CES technology is capable of detecting various of known variant types, including SNVs, copy number variations (CNVs), small insertion and deletions (indels), chromosome aneuploidy, uniparental disomy (UPD), and mosaicism (Feng et al., 2015; Yu et al., 2016).

\section{Variant Analysis and Interpretation}

Raw-image data conversion and demultiplexing were performed following Illumina's primary data analysis pipeline using CASAVA version 2.0 (Illumina). Low-quality reads (Phred score $<\mathrm{Q} 20$ ) were removed before demultiplexing.
Sequences were aligned to the hg19 reference genome by NextGENe (SoftGenetics, State College, PA) using the recommended standard settings for single-nucleotide variant and insertion/deletion discovery. The variants with minor allele frequency $(\mathrm{MAF})>1 \%$ in the Asian population were filtered out. Variant annotation was further confirmed through literature and population databases, including 1000 Genomes, dbSNP, GnomAD, Clinvar, HGMD, and OMIM. Genetic evaluation of pathogenicity of candidate gene variants was performed using multiple computational algorithms, including SIFT (Craig Venter Institute), Polyphen-2 (Harvard University), and MutationTaster (NeuroCure Cluster of Excellence). The interpretation of variants was performed according to the American College of Medical Genetics (ACMG) guidelines (Richards et al., 2015).

\section{mtDNA Sequencing}

The whole mitochondrial genome sequencing (16,569 bp) was performed using long-PCR followed by next generation sequencing (Yang et al., 2020). The mtDNA was extracted from peripheral blood from patients and maternal samples using a commercial DNA extraction kit (Mike Bio, China) according to the manufacturer's instructions. The whole mitochondrial genome was amplified using long-range PCR, and disrupted by sonication using a Q800R Sonicator (Qsonica). High throughput sequencing was performed using a NextSeq 500 sequencer (Illumina, United States). The average depth of sequencing was at least $5000 \times$. After the data was obtained, the bioinformatics analysis of the gene sequence was performed to confirm the locus information of the pathogenic genes. This assay detects single nucleotide variants with heteroplasmy $>2 \%$. 


\section{Pediatric patients with a severe and/or progressive disease who met with the enrollment criteria were detected by rapid CES for a trio analysis as a first-tier test $(n=40)$}

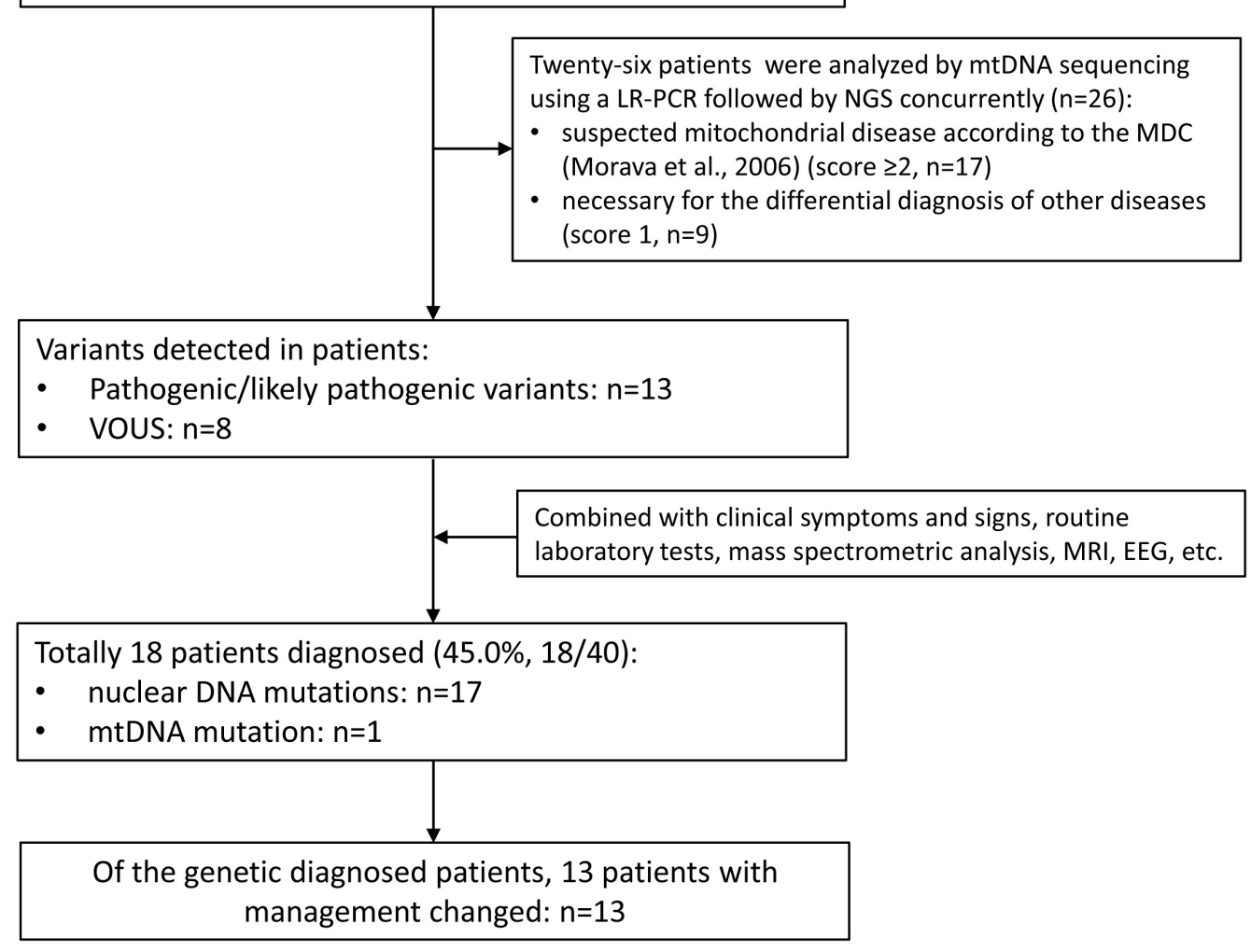

FIGURE 1 | The flowchart of the genetic diagnosis in the 40 pediatric patients. CES, clinical exome sequencing; MDC, mitochondrial disease criteria; VOUS, variants of unknown significance.

\section{RESULTS}

\section{Clinical Characteristics}

The clinical characteristics of the patients who went through the rapid CES and mtDNA sequencing were summarized in Table 1. Our cohort comprised of 24 males and 16 females with a median age of 4.5 months (range, 6 days-11 years) at the time rapid CES was performed, including 6 newborns less than one month old, 25 infants aged 1-12 months old, and 9 young children aged 111 years old. Among the patients, there were 18 patients in the NICU, 11 patients in the PICU, and 11 patients in other pediatric wards. Regarding the family history, 2 patients were twin sisters with the same phenotype (patient ID: P14, P15). All the patients were born to non-consanguineous parents, and none had an affected parent.

\section{Genetic Diagnosis, TAT}

According to the genetic test results and clinical phenotypes, a genetic diagnosis was made in 18 patients (18/40, 45.0\%); three cases with de novo autosomal dominant variants (involving the genes KRAS, SCN5A, and WT1), nine cases with homozygous or compound heterozygous variants (involving the genes IL10RA,
CD19, SLC37A4, UPB1, GCDH, AMPD1, VWF, and LHX3), three cases with hemizygous variants inherited from mother (including the genes HSD17B10, SLC9A6, and NAA10), two cases with heterozygous variants inherited from either parent $(A B C C 8$ and MYH7 genes), and one case with a mtDNA mutation (MT-ND6 gene) (Table 2). The variation types detected in the 18 patients included missense and frameshift mutations, while zero clinically significant CNVs were detected in this study.

The rapid trio CES combined with mtDNA sequencing strategy enabled us to obtain a median TAT of 5 days from blood sample receipt to test reporting to ordering clinician (range 39 days). In three severely ill patients, the TAT of rapid CES and mtDNA sequencing was 3 days. For de novo variants or uncertain variants that suspected homologous interference needed to be confirmed by Sanger sequencing, thus, the final report was issued 2 days thereafter.

\section{Impact of the Rapid Genetic Diagnosis on Clinical Procedures}

Thirteen patients that were diagnosed had an available medical treatment option and resulted in a positive outcome. For example, a female infant (ID: P38) was admitted to the PICU 
TABLE 2 | Clinical features and genetic outcome of the 18 patients diagnosed.

\begin{tabular}{|c|c|c|c|c|c|c|c|c|c|c|c|}
\hline $\begin{array}{l}\text { Cases } \\
\text { diagnosed in } \\
\text { the category }\end{array}$ & Patient ID & $\begin{array}{l}\text { Sex/Age } \\
\text { at testing }\end{array}$ & Clinical features & $\begin{array}{l}\text { Gene/ } \\
\text { OMIM ID }\end{array}$ & $\begin{array}{l}\text { Ref. Seq } \\
\text { (hg19) }\end{array}$ & $\begin{array}{l}\text { Variants/ } \\
\text { Transcript_ID }\end{array}$ & $\begin{array}{l}\text { Variant } \\
\text { classifi- } \\
\text { cation }\end{array}$ & $\begin{array}{l}\text { Clinically } \\
\text { reported } \\
\text { (Yes/No) }\end{array}$ & Zygosity & $\begin{array}{l}\text { Inheri- } \\
\text { tance } \\
\text { pattern }\end{array}$ & Disease \\
\hline \multirow[t]{6}{*}{$\begin{array}{l}\text { Metabolic- } \\
\text { related } \\
\text { diseases: } n=6\end{array}$} & P8 & $\mathrm{M} / 3 \mathrm{~m}$ & $\begin{array}{l}\text { Mass spectrometry showed } \\
\text { multiple metabolite } \\
\text { abnormalities, } \\
\text { hyperlactacidemia }\end{array}$ & $\begin{array}{l}\text { SLC37A4 } \\
(602671)\end{array}$ & 11:118898518 & $\begin{array}{l}\text { c.446G > A } \\
\text { (p.G149E) } \\
\text { [NM_001164278] }\end{array}$ & LP & Yes & Hom & $\begin{array}{l}\text { AR; } \\
\text { Inherited } \\
\text { from both } \\
\text { parents }\end{array}$ & $\begin{array}{l}\text { Glycogen storage disease } \\
\text { type Ic } \\
\text { (MIM: 232240) }\end{array}$ \\
\hline & $\mathrm{P} 13^{\#}$ & $\mathrm{M} / 18 \mathrm{~m}$ & $\begin{array}{l}\text { Psychomotor retardation, } \\
\text { developmental regression } \\
\text { at } 10 \text { months old, } \\
\text { strabismus, nystagmus, } \\
\text { bucking, feeding difficulties, } \\
\text { hypotonia }\end{array}$ & MT-ND6 & M:14430 & $\begin{array}{l}\mathrm{m} .14430 \mathrm{~A}>\mathrm{G} \\
\text { (p.W82R) } \\
\text { [NC_012920] }\end{array}$ & LP & Yes & $\begin{array}{l}\text { mtDNA } \\
\text { mutation } \\
\text { load of } \\
91.3 \%\end{array}$ & $\begin{array}{l}\text { Maternal } \\
\text { inheritance } \\
(7.9 \%)\end{array}$ & $\begin{array}{l}\text { Leigh syndrome } \\
\text { (MIM: 256000) }\end{array}$ \\
\hline & P24 & $\mathrm{M} / 1 \mathrm{~m}$ & $\begin{array}{l}\text { Respiratory distress } \\
\text { syndrome, mass } \\
\text { spectrometry showed } \\
\text { elevated } \beta \text {-UP and } \beta \text {-UIB }\end{array}$ & $\begin{array}{l}\text { UPB1 } \\
\text { (606673) }\end{array}$ & 22:24919647 & $\begin{array}{l}\text { c.977G }>A \\
\text { (p.R326Q) } \\
{[\text { NM_016327] }}\end{array}$ & LP & Yes & Hom & $\begin{array}{l}\text { AR; } \\
\text { Inherited } \\
\text { from both } \\
\text { parents }\end{array}$ & $\begin{array}{l}\text { Beta-ureidopropionase } \\
\text { deficiency } \\
\text { (MIM: 613161) }\end{array}$ \\
\hline & P28\# & $\mathrm{M} / 7 \mathrm{~d}$ & $\begin{array}{l}\text { Fetal macrosomia, } \\
\text { hypoglycemia }\end{array}$ & $\begin{array}{l}A B C C 8 \\
(600509)\end{array}$ & 11:17428474 & $\begin{array}{l}\text { c.3250_3252 } \\
\text { delins13 } \\
\text { (p.T1042Qfs* 75) } \\
\text { [NM_000352] }\end{array}$ & LP & No & Het & $\begin{array}{l}\text { AD/AR; } \\
\text { Inherited } \\
\text { from father }\end{array}$ & $\begin{array}{l}\text { Familial hyperinsulinemic } \\
\text { hypoglycemia } \\
\text { (MIM: 256450) }\end{array}$ \\
\hline & P36\# & $\mathrm{M} / 11 \mathrm{~m}$ & $\begin{array}{l}\text { Sudden irritability and } \\
\text { dyspnea, } \\
\text { hyperlactacidemia, } \\
\text { respiratory and circulatory } \\
\text { failure }\end{array}$ & $\begin{array}{l}\text { HSD17B10 } \\
(300256)\end{array}$ & $X: 53458838$ & $\begin{array}{l}\text { c.503A }>\text { G } \\
(p . Y 168 C) \\
{\left[N M \_004493\right]}\end{array}$ & vous & No & Hem & $\begin{array}{l}\mathrm{XL} ; \\
\text { Inherited } \\
\text { from } \\
\text { mother }\end{array}$ & $\begin{array}{l}\text { HSD10 mitochondrial } \\
\text { disease } \\
\text { (MIM: 300438) }\end{array}$ \\
\hline & P38 $\#$ & $\mathrm{~F} / 6 \mathrm{~m}$ & $\begin{array}{l}\text { Obnubilation, hypotonia, } \\
\text { infantile encephalopathy, } \\
\text { brain MRI showed } \\
\text { abnormal signals in bilateral } \\
\text { basal ganglia and white } \\
\text { matter, and atrophy of the } \\
\text { temporal lobes }\end{array}$ & $\begin{array}{l}\text { GCDH } \\
(608801)\end{array}$ & $\begin{array}{l}\text { 19:13004345; } \\
\text { 19:13007744 }\end{array}$ & $\begin{array}{l}\text { c.383G > A } \\
\text { (p.R128Q); } \\
\text { c.873delC } \\
\text { (p.N291Kfs* 41) } \\
{[\text { NM_000159] }}\end{array}$ & $\begin{array}{l}\text { LP; } \\
\text { LP }\end{array}$ & $\begin{array}{l}\text { Yes; } \\
\text { No }\end{array}$ & $\begin{array}{l}\text { Compound } \\
\text { Het }\end{array}$ & $\begin{array}{l}\text { AR; } \\
\text { Inherited } \\
\text { from both } \\
\text { parents }\end{array}$ & $\begin{array}{l}\text { Glutaricaciduria type I } \\
\text { (MIM: 231670) }\end{array}$ \\
\hline \multirow[t]{2}{*}{$\begin{array}{l}\text { Immunodefi- } \\
\text { ciency } \\
\text { diseases: } n=4\end{array}$} & P1 & $\mathrm{F} / 7 \mathrm{~m}$ & $\begin{array}{l}\text { Diarrhea, perianal abscess, } \\
\text { pyrexia, skin eczema }\end{array}$ & $\begin{array}{l}\text { IL10RA } \\
\text { (146933) }\end{array}$ & 11:117860269 & $\begin{array}{l}\text { c.301C > T } \\
\text { (p.R101W) } \\
\text { [NM_001558] }\end{array}$ & LP & Yes & Hom & $\begin{array}{l}\text { AR; } \\
\text { Inherited } \\
\text { from both } \\
\text { parents }\end{array}$ & $\begin{array}{l}\text { Early-onset inflammatory } \\
\text { bowel disease } \\
\text { (MIM: 613148) }\end{array}$ \\
\hline & P5 & $\mathrm{F} / 10 \mathrm{~m}$ & $\begin{array}{l}\text { Diarrhea, pyrexia, } \\
\text { splenomegaly, rash, } \\
\text { hyperbilirubinemia }\end{array}$ & $\begin{array}{l}\text { KRAS } \\
(190070)\end{array}$ & 12:25378647 & $\begin{array}{l}\text { c.351A > C } \\
(\text { p.K117N) } \\
\text { [NM_033360] }\end{array}$ & LP & No & Het & $\begin{array}{l}\mathrm{AD} ; \mathrm{De} \\
\text { novo }\end{array}$ & $\begin{array}{l}\text { RAS-associated } \\
\text { autoimmune } \\
\text { leukoproliferative disorder } \\
\text { (MIM: 614470) }\end{array}$ \\
\hline
\end{tabular}


TABLE 2 | Continued

\begin{tabular}{|c|c|c|c|c|c|c|c|c|c|c|c|}
\hline $\begin{array}{l}\text { Cases } \\
\text { diagnosed in } \\
\text { the category }\end{array}$ & Patient ID & $\begin{array}{l}\text { Sex/Age } \\
\text { at testing }\end{array}$ & Clinical features & $\begin{array}{l}\text { Gene/ } \\
\text { OMIM ID }\end{array}$ & $\begin{array}{l}\text { Ref. Seq } \\
\text { (hg19) }\end{array}$ & $\begin{array}{l}\text { Variants/ } \\
\text { Transcript_ID }\end{array}$ & $\begin{array}{l}\text { Variant } \\
\text { classifi- } \\
\text { cation }\end{array}$ & $\begin{array}{l}\text { Clinically } \\
\text { reported } \\
\text { (Yes/No) }\end{array}$ & Zygosity & $\begin{array}{l}\text { Inheri- } \\
\text { tance } \\
\text { pattern }\end{array}$ & Disease \\
\hline & $\begin{array}{l}\mathrm{P} 14^{\#} \text { and } \\
\mathrm{P} 15^{\#} \\
\text { (twins) }\end{array}$ & $\mathrm{F} / 6 \mathrm{~m}$ & $\begin{array}{l}\text { Severe infection, electrolyte } \\
\text { disturbance, severe } \\
\text { metabolic alkalosis }\end{array}$ & $\begin{array}{l}\text { CD19 } \\
(107265)\end{array}$ & $\begin{array}{l}\text { 16:28943883; } \\
\text { 16:28947515 }\end{array}$ & $\begin{array}{l}\text { c.305C > } \\
\text { (p.P102R); } \\
\text { c.988A > G } \\
\text { (p.T330A) } \\
\text { [NM_001178098] }\end{array}$ & $\begin{array}{l}\text { VOUS; } \\
\text { VOUS }\end{array}$ & $\begin{array}{l}\text { No; } \\
\text { No }\end{array}$ & $\begin{array}{l}\text { Compound } \\
\text { Het }\end{array}$ & $\begin{array}{l}\text { AR; } \\
\text { Inherited } \\
\text { from both } \\
\text { parents }\end{array}$ & $\begin{array}{l}\text { Common variable } \\
\text { immunodeficiency } \\
\text { (MIM: 613493) }\end{array}$ \\
\hline \multirow[t]{2}{*}{$\begin{array}{l}\text { Cardiovascular } \\
\text { diseases: } n=2\end{array}$} & P18 & $M / 5 y$ & $\begin{array}{l}\text { Malignant arrhythmia: } \\
\text { ventricular tachycardia, } \\
\text { ventricular fibrillation, atrial } \\
\text { tachycardia, atrial fibrillation }\end{array}$ & $\begin{array}{l}\text { SCN5A } \\
(600163)\end{array}$ & 3:38592971 & $\begin{array}{l}\text { c. } 4892 G>A \\
\text { (p.G1631D) } \\
\text { [NM_001099404] }\end{array}$ & LP & Yes & Het & $\begin{array}{l}\mathrm{AD} ; \mathrm{De} \\
\text { novo }\end{array}$ & $\begin{array}{l}\text { Familial atrial fibrillation } \\
\text { (MIM: 614022) }\end{array}$ \\
\hline & $\mathrm{P} 21^{\#}$ & $\mathrm{~F} / 4 \mathrm{~m}$ & $\begin{array}{l}\text { Poor appetite, enlarged left } \\
\text { ventricle, reduced systolic } \\
\text { and diastolic function }\end{array}$ & $\begin{array}{l}\text { MYH7 } \\
(160760)\end{array}$ & 14:23902427 & $\begin{array}{l}\text { c.211G > } \\
\text { (p.V71M) } \\
\text { [NM_000257] }\end{array}$ & LP & No & Het & $\begin{array}{l}\text { AD; } \\
\text { Inherited } \\
\text { from } \\
\text { mother }\end{array}$ & $\begin{array}{l}\text { Cardiomyopathy dilated } \\
\text { type 1S } \\
\text { (MIM: 613426) }\end{array}$ \\
\hline \multirow[t]{2}{*}{$\begin{array}{l}\text { Neuromuscular } \\
\text { diseases: } n=2\end{array}$} & P23 ${ }^{\#}$ & $\mathrm{M} / 9 \mathrm{~m}$ & $\begin{array}{l}\text { Motor retardation, } \\
\text { hypotonia and weakness of } \\
\text { the whole body }\end{array}$ & $\begin{array}{l}\text { AMPD1 } \\
(102770)\end{array}$ & $\begin{array}{l}\text { 1:115223070; } \\
\text { 1:115220125 }\end{array}$ & $\begin{array}{l}\text { c.676G > A } \\
(\text { p.D226N); } \\
\text { c.1334C > T } \\
(\text { p.A445V) } \\
\text { [NM_000036] }\end{array}$ & $\begin{array}{l}\text { VOUS; } \\
\text { VOUS }\end{array}$ & $\begin{array}{l}\text { No; } \\
\text { No }\end{array}$ & $\begin{array}{l}\text { Compound } \\
\text { Het }\end{array}$ & $\begin{array}{l}\text { AR; } \\
\text { Inherited } \\
\text { from both } \\
\text { parents }\end{array}$ & $\begin{array}{l}\text { Myoadenylate deaminase } \\
\text { deficiency myopathy } \\
\text { (MIM: 615511) }\end{array}$ \\
\hline & P26 ${ }^{\#}$ & $\mathrm{M} / 2 \mathrm{~m}$ & $\begin{array}{l}\text { Seizures, head CT showed } \\
\text { bilateral symmetrical } \\
\text { low-density images in } \\
\text { parietal lobe }\end{array}$ & $\begin{array}{l}\text { SLC9A6 } \\
(300231)\end{array}$ & X:135080641 & $\begin{array}{l}c .604 A>G \\
(p . R 202 G) \\
\text { [NM_001042537] }\end{array}$ & VOUS & No & Hem & $\begin{array}{l}\mathrm{XL} ; \\
\text { Inherited } \\
\text { from } \\
\text { mother }\end{array}$ & $\begin{array}{l}\text { Christianson syndrome } \\
\text { (MIM: 300243) }\end{array}$ \\
\hline \multirow[t]{2}{*}{$\begin{array}{l}\text { Multi-system } \\
\text { diseases: } n=2\end{array}$} & P16 & $\mathrm{M} / 2 \mathrm{~m}$ & $\begin{array}{l}\text { Small for gestational age } \\
\text { infant, cyanoderma, } \\
\text { hyoxemia, } \\
\text { hyperbilirubinemia, special } \\
\text { face }\end{array}$ & $\begin{array}{l}\text { NAA10 } \\
(300013)\end{array}$ & X:153199841 & $\begin{array}{l}\text { c.109T > C } \\
\text { (p.S37P) } \\
\text { [NM_003491] }\end{array}$ & LP & Yes & Hem & $\begin{array}{l}\mathrm{XL} \text {; } \\
\text { Inherited } \\
\text { from } \\
\text { mother }\end{array}$ & $\begin{array}{l}\text { Ogden syndrome } \\
\text { (MIM: 300855) }\end{array}$ \\
\hline & P39 & $M / 23 d$ & $\begin{array}{l}\text { Low response, } \\
\text { hypospadias, short penis, } \\
\text { systemic pitting edema }\end{array}$ & $\begin{array}{l}\text { WT1 } \\
(607102)\end{array}$ & 11:32413565 & $\begin{array}{l}\text { c. } 1385 G>A \\
\text { (p.R462Q) } \\
\text { [NM_024426] }\end{array}$ & LP & Yes & Het & $\begin{array}{l}\mathrm{AD} ; \mathrm{De} \\
\text { novo }\end{array}$ & $\begin{array}{l}\text { Denys-Drash syndrome } \\
\text { (MIM: 194080) }\end{array}$ \\
\hline $\begin{array}{l}\text { Hematological } \\
\text { diseases: } n=1\end{array}$ & P20 \# & $F / 6 d$ & $\begin{array}{l}\text { Respiratory distress, } \\
\text { thrombocytopenia, } \\
\text { coagulation abnormalities }\end{array}$ & $\begin{array}{l}\text { VWF } \\
(613160)\end{array}$ & $\begin{array}{l}\text { 12:6132811; } \\
\text { 12:6103733 }\end{array}$ & $\begin{array}{l}\text { c.3365C > T } \\
\text { (p.T1122M); } \\
\text { c.6104G > A } \\
\text { (p.G2035D) } \\
\text { [NM_000552] }\end{array}$ & $\begin{array}{l}\text { LP; } \\
\text { LP }\end{array}$ & $\begin{array}{l}\text { Yes; } \\
\text { Yes }\end{array}$ & $\begin{array}{l}\text { Compound } \\
\text { Het }\end{array}$ & $\begin{array}{l}\text { AR; } \\
\text { Inherited } \\
\text { from both } \\
\text { parents }\end{array}$ & $\begin{array}{l}\text { Von Willebrand disease } \\
\text { type } 3 \\
\text { (MIM: 277480) }\end{array}$ \\
\hline $\begin{array}{l}\text { Endocrine } \\
\text { diseases: } n=1\end{array}$ & P22 ${ }^{\#}$ & $M / 11 d$ & $\begin{array}{l}\text { Small head circumference, } \\
\text { hypoglycemia, } \\
\text { hypothyroidism, left } \\
\text { cryptorchidism, deafness }\end{array}$ & $\begin{array}{l}\text { LHX3 } \\
(600577)\end{array}$ & $\begin{array}{l}\text { 9:139090825; } \\
\text { 9:139090762 }\end{array}$ & $\begin{array}{l}\text { c.550T > C } \\
(\text { p.S184P); } \\
\text { c.613G >C } \\
\text { (p.V205L) } \\
\text { [NM_014564] }\end{array}$ & $\begin{array}{l}\text { LP; } \\
\text { LP }\end{array}$ & $\begin{array}{l}\text { No; } \\
\text { No }\end{array}$ & $\begin{array}{l}\text { Compound } \\
\text { Het }\end{array}$ & $\begin{array}{l}\text { AR; } \\
\text { Inherited } \\
\text { from both } \\
\text { parents }\end{array}$ & $\begin{array}{l}\text { Combined pituitary } \\
\text { hormone deficiency type } 3 \\
\text { (MIM: 221750) }\end{array}$ \\
\hline
\end{tabular}

\#, CES and mtDNA sequencing; M, male; F, female; d, day; m, month; y, year; LP, Likely pathogenic; VOUS, various of unknown significance; Hom, homozygote; Het, heterozygote; Hem, hemizygote; mtDNA, mitochondrial $D N A ; A R$, autosomal recessive; $A D$, autosomal dominant; $X L, X$-linked. 
at 6 months of age with irritability, dystonia, and atrophy of the temporal lobes. MRI results showed abnormal bilateral basal ganglia and white matter signal, and atrophy of the temporal lobes. The rapid CES and mtDNA sequencing were applied to the patient concurrently, and test reports were issued within 5 days. CES identified compound heterozygous mutations (p.R128Q and p.N291Kfs $* 41$ ) in the GCDH gene (OMIM: 608801), then the patient was diagnosed with glutaric aciduria type I. She was immediately treated by a low-lysine diet and carnitine supplementation according to the guidelines for the management of glutaric aciduria type I (Heringer et al., 2010) and were considered for further long-term follow-up as indicated.

A male infant (ID: P8) was admitted to the PICU at 3 months of age with lactic acidosis and hypoglycemia. A molecular diagnosis of glycogen storage disease Ib caused by compound heterozygous mutations (p.G149E and p.G149E) in the SLC37A4 gene (OMIM: 602671) was detected by rapid CES. He was immediately treated by continuous night-time intragastric glucose infusion through a nasogastric tube, frequent feedings during the daytime with high complexity carbohydrates, and oral administration of uncooked cornstarch was also recommended after 6 months old according to the guidelines for the management of glycogen storage disease type I (Kishnani et al., 2014). These treatments successfully made the boy maintain a normal blood glucose concentration and corrected most of the other metabolic abnormalities.

Patient 18 (ID: P18) was admitted to the PICU at 4 years old with malignant arrhythmia including ventricular tachycardia, ventricular fibrillation, atrial tachycardia and atrial fibrillation. A de novo heterozygous mutation (p.G1631D) in the SCN5A gene (OMIM: 600163) was detected by rapid CES. The SCN5A gene defects have been associated with Brugada syndrome, familial atrial or ventricular fibrillation, and long QT syndrome. He was given beta blockers (propranolol), and cessation of symptoms was observed.

Patient 28 (ID: P28) was admitted to the NICU at birth with hypoglycemia, hyperinsulinism, and adrenal hyperplasia. He underwent rapid CES and mtDNA sequencing, and a diagnosis of "familial hyper insulinemic hypoglycemia" was made by detecting a heterozygous mutation (p.T1042Qfs * 75) in the ABCC8 gene (OMIM: 600509) inherited from the father. He was given diazoxide therapy combined with frequent feedings, and showed an excellent response to this treatment with substantially improved plasma glucose concentrations.

\section{DISCUSSION}

Of the known genetic diseases according to the database of OMIM, the majority predominantly affect infants and children, which are the leading cause of infant mortality and pediatric hospital admissions. There is evidence that a significant number of genetic diseases present with clinical phenotypes during the first 28 days or the first year of life. However, precise early diagnosis of genetic diseases in these pediatric patients is very challenging, because the full clinical symptoms may not be evident in newborns or infants. Many studies have shown that genome-wide sequencing based on NGS technologies are powerful approaches for genetic testing in clinical settings. In recent years, rapid WGS and WES have been effectively used for genetic analysis in critically ill pediatric patients with suspected genetic disorders in the PICU/NICU, with a diagnosis rate ranging from 30 to $72.2 \%$ in different pediatric patient cohorts (Bourchany et al., 2017; van Diemen et al., 2017; Farnaes et al., 2018; Brunelli et al., 2019; Elliott et al., 2019; Sanford et al., 2019; Smigiel et al., 2020; Wang et al., 2020). In the present study, we applied rapid CES in 40 patients with severe and/or progressive diseases (most in NICU/PICU), as well as mtDNA sequencing in 26 patients that could not exclude mitochondrial disorders. Among the 40 patients, 17 patients were diagnosed as monogenetic disorders caused by mutations on nuclear genome, and one patient was diagnosed as a mitochondrial disorder caused by a mtDNA mutation, with an overall yield rate of $45.0 \%(18 / 40)$.

Inborn errors of metabolism (IEM) are a group of rare genetic diseases that generally result from impaired enzyme activities in specific metabolic pathways by genetic mutations. Recent articles reported more than 1015 known IEM, of which mitochondrial disorders are the most common group (about 23\%) of inherited metabolic diseases (Ferreira et al., 2019; Ismail et al., 2019). To date, pathogenic variants in more than 300 nuclear genes and 37 mitochondrial genes are known to cause mitochondrial diseases (Schon et al., 2020). The genetic basis of mitochondrial disorders is very complex, and Mendelian inheritance and maternal inheritance patterns can all lead to both familial and sporadic cases. In this study, IEM was found in 6 patients, including two cases with mitochondrial disorders. A recent study on 18 unrelated infants in an ICU has also shown that mitochondrial disorders identified by rapid WES are the main cause of IEM (Smigiel et al., 2020). The prevalence of mtDNA mutation diseases is estimated to be at least 1 in 5000 (Skladal et al., 2003; Schaefer et al., 2004), and earlyonset disease $(<1$ year) has been reported in many studies (Honzik et al., 2012; Bannwarth et al., 2013; Akesson et al., 2019; Imai-Okazaki et al., 2019). However, mtDNA sequencing is not routinely available in current rapid genetic diagnosis programs, which may fail to diagnose mtDNA mutation-associated diseases. In addition to nuclear genome, WGS can also provide a relatively good coverage (approximately 1000-2000x) of the mitochondrial genome to identify mtDNA variant, specifically a single nucleotide variant (Saunders et al., 2012; Petrikin et al., 2015; Brockhage et al., 2018; Husami et al., 2020; Riley et al., 2020), while ES may detect single nucleotide variants in mtDNA (Pronicka et al., 2016; Puusepp et al., 2018). The mitochondrial genome may be impacted by nuclear mitochondrial DNA segments (NUMTs), resulting in false positive mtDNA variants, failure to distinguish between heteroplasmy and homoplasmy, and inaccurate quantification of mtDNA variants (Ramos et al., 2013; Puertas and Gonzalez-Sanchez, 2020). In this study, the whole mitochondrial genome sequence was performed using a long-PCR based NGS method (Saunders et al., 2012; Yang et al., 2020). An 18-month-old male patient (ID: P13) with early-onset psychomotor retardation and developmental regression had a missense mutation (m.14430A > G, p.W82R) with mutation load 
of $91.3 \%$ in the MT-ND6 gene that was diagnosed with Leigh syndrome.

The clinical diagnosis of mitochondrial diseases present challenges for physicians, starting with when and how to investigate a suspected mitochondrial disorder. When a patient is clinically suspected of mitochondrial disease, the traditional approach is to exclude other common or metabolic disorders followed testing affected tissue with biochemical and histochemical assays to reach a final diagnosis. However, this procedure is expensive, invasive, time-consuming, and often no definitive diagnosis is reached. The modified mitochondrial disease criteria (MDC) in 2006 classified patients as having a "possible," "probable," or "definite" mitochondrial disorder according to score based on clinical signs and symptoms, metabolic/imaging tests, and morphology (Morava et al., 2006). With the advent of next-generation sequencing, exome sequencing was used to identify genetic defects in suspected mitochondrial patients. Wortmann et al. reported that a molecular diagnosis was achieved in $57 \%$ of the subgroup of patients with the highest suspicion for a mitochondrial disease, while several genes associated with neuromuscular disorders were identified in the subgroup of patients with the lowest suspicion for a mitochondrial disorder (Wortmann et al., 2015). The application of WES in patients suspected with mitochondrial disease indicated that nuclear DNA variants are more common, single nucleotide variants in mtDNA may also be detected, and that a significant proportion of non-mitochondrial disorders caused by other variants were also found (Pronicka et al., 2016; Puusepp et al., 2018). In a multicenter cohort of 136 patients clinically diagnosed with mitochondrial disorders, mtDNA restriction fragment length polymorphism (RFLP) and/or exome sequencing (including WES or gene panels) were performed to confirm the genetic etiology, and the results showed that $51 \%$ had a maximal mitochondrial score (8/8) predicting a definite mitochondrial disorder, $33 \%$ had a probable mitochondrial disorder (5-7/8), and $16 \%$ had a possible mitochondrial disorder $(2-4 / 8)$ (Witters et al., 2018). Previous studies indicated that WES analysis has been successfully implemented as a first-tier test for mitochondrial disorders as well as for the mimicking disorders in clinical practice, and clinical and biochemical phenotyping is essential for successful application of WES to diagnose these patients. In view of the broad phenotypic and genotypic heterogeneity of mitochondrial disorders, we suggest that exome sequencing in combination with mtDNA sequencing is applied to identify genetic defects for all patients that could not exclude a mitochondrial disorder according to the MDC.

Early diagnosis of suspected genetic disorders can help guide clinical management, greatly improve patient outcomes, and reduce health care cost. In this study, the average turnaround time to reach a diagnosis was 5 days, with a range of 3-9 days, which is significantly faster than routine exome sequencing. Currently, rapid whole exome and target sequencing can deliver a molecular diagnosis for patients with the TAT of 1-2 weeks, while the TAT of rWGS was generally less than one week even within $24 \mathrm{~h}$, positively changing the medical management. In this study, 13 patients that were diagnosed had an available medical treatment and resulted in a positive outcome. We have not analyzed the economic benefits and issue, but it is highly possible that rapid CES and mtDNA sequencing as a first-tier test is time and cost-effective by significantly shortened the time of hospitalization for patients in NICU/PICU.

In conclusion, rapid CES proved to be an effective diagnostic tool for critically ill pediatric patients with suspected genetic disorders, which prevented a range of unnecessary investigations and guided the appropriate treatment. We have demonstrated the feasibility and utility of incorporating rapid mitochondrial genome sequencing combined with ES in patients whose diagnosis or differential diagnosis includes mitochondrial disorders.

\section{DATA AVAILABILITY STATEMENT}

The datasets for this article are not publicly available due to concerns regarding participant/patient anonymity. Requests to access the datasets should be directed to the corresponding authors.

\section{ETHICS STATEMENT}

The studies involving human participants were reviewed and approved by The ethics committee of Zhujiang Hospital. Written informed consent was obtained from the individual(s)' and minor(s)' legal guardian/next of kin, for the publication of any potentially identifiable images or data included in this article.

\section{AUTHOR CONTRIBUTIONS}

$\mathrm{XO}, \mathrm{BW}$, and $\mathrm{VZ}$ designed the study and drafted the initial manuscript. YZ, LjZ, JL, TZ, HH, LL, LqZ, SZ, PX, ZB, and CW supervised all aspects of data collection, analysis, and manuscript production. L-JW and JW reviewed and revised the manuscript. All authors reviewed and revised the manuscript and approved the final manuscript as submitted.

\section{FUNDING}

This work was supported by Major Medical Collaboration and Innovation Program of Guangzhou Science Technology and Innovation Commission (Grant Nos. 201604020020 and 201604020009), a Science and Technology Planning Project of Guangzhou (2018-1202-SF-0019) to VZ. This work was also supported by Scientific Research Foundation for Postdoctoral and research grants from National Key Research and Development Projects of China (2016YFC1306601), the National Natural Science Foundation of China (81471292, U1603281, U1503222, 81430021, and 81603681), the Science and Technology Project of Guangdong Province (2015A030311021 and 2016A020215201), a Science and Technology Planning Project of Guangzhou (201504281820463 and 2018-1202-SF0019), and an international project of Science and Technology for Guangdong (2016A050502025) to PX. 


\section{REFERENCES}

Akesson, L. S., Eggers, S., Love, C. J., Chong, B., Krzesinski, E. I., Brown, N. J., et al. (2019). Early diagnosis of Pearson syndrome in neonatal intensive care following rapid mitochondrial genome sequencing in tandem with exome sequencing. Eur. J. Hum. Genet. 27, 1821-1826. doi: 10.1038/s41431-0190477-3

Australian Genomics Health Alliance Acute Care Flagship, Lunke, S., Eggers, S., Wilson, M., Patel, C., Barnett, C. P., et al. (2020). Feasibility of UltraRapid Exome Sequencing in Critically Ill Infants and Children With Suspected Monogenic Conditions in the Australian Public Health Care System. JAMA 323, 2503-2511. doi: 10.1001/jama.2020.7671

Bannwarth, S., Procaccio, V., Lebre, A. S., Jardel, C., Chaussenot, A., Hoarau, C., et al. (2013). Prevalence of rare mitochondrial DNA mutations in mitochondrial disorders. J. Med. Genet. 50, 704-714. doi: 10.1136/jmedgenet-2013-101604

Bourchany, A., Thauvin-Robinet, C., Lehalle, D., Bruel, A. L., Masurel-Paulet, A., Jean, N., et al. (2017). Reducing diagnostic turnaround times of exome sequencing for families requiring timely diagnoses. Eur. J. Med. Genet. 60, 595-604. doi: 10.1016/j.ejmg.2017.08.011

Brockhage, R., Slone, J., Ma, Z., Hegde, M. R., Valencia, C. A., and Huang, T. (2018). Validation of the diagnostic potential of mtDNA copy number derived from whole genome sequencing. J. Genet. Genomics [Epub Online ahead of print]. doi: 10.1016/j.jgg.2018.06.001

Brunelli, L., Jenkins, S. M., Gudgeon, J. M., Bleyl, S. B., Miller, C. E., Tvrdik, T., et al. (2019). Targeted gene panel sequencing for the rapid diagnosis of acutely ill infants. Mol. Genet. Genomic Med. 7:e00796. doi: 10.1002/mgg3.796

Cakici, J. A., Dimmock, D. P., Caylor, S. A., Gaughran, M., Clarke, C., Triplett, C., et al. (2020). A Prospective Study of Parental Perceptions of Rapid WholeGenome and -Exome Sequencing among Seriously Ill Infants. Am. J. Hum. Genet. 107, 953-962. doi: 10.1016/j.ajhg.2020.10.004

Clark, M. M., Hildreth, A., Batalov, S., Ding, Y., Chowdhury, S., Watkins, K., et al. (2019). Diagnosis of genetic diseases in seriously ill children by rapid wholegenome sequencing and automated phenotyping and interpretation. Sci. Transl. Med. 11:eaat6177. doi: 10.1126/scitranslmed.aat6177

Dimmock, D. P., Clark, M. M., Gaughran, M., Cakici, J. A., Caylor, S. A., Clarke, C., et al. (2020). An RCT of Rapid Genomic Sequencing among Seriously Ill Infants Results in High Clinical Utility, Changes in Management, and Low Perceived Harm. Am. J. Hum. Genet. 107, 942-952. doi: 10.1016/j.ajhg.2020.10.003

Elliott, A. M., du Souich, C., Lehman, A., Guella, I., Evans, D. M., Candido, T., et al. (2019). RAPIDOMICS: rapid genome-wide sequencing in a neonatal intensive care unit-successes and challenges. Eur. J. Pediatr. 178, 1207-1218. doi: 10.1007/s00431-019-03399-4

Farnaes, L., Hildreth, A., Sweeney, N. M., Clark, M. M., Chowdhury, S., Nahas, S., et al. (2018). Rapid whole-genome sequencing decreases infant morbidity and cost of hospitalization. NPJ Genom. Med. 3:10. doi: 10.1038/s41525-018-0049-4

Feng, Y., Chen, D., Wang, G. L., Zhang, V. W., and Wong, L. J. (2015). Improved molecular diagnosis by the detection of exonic deletions with target gene capture and deep sequencing. Genet. Med. 17, 99-107. doi: 10.1038/gim. 2014.80

Ferreira, C. R., van Karnebeek, C. D. M., Vockley, J., and Blau, N. (2019). A proposed nosology of inborn errors of metabolism. Genet. Med. 21, 102-106. doi: 10.1038/s41436-018-0022-8

French, C. E., Delon, I., Dolling, H., Sanchis-Juan, A., Shamardina, O., Megy, K., et al. (2019). Whole genome sequencing reveals that genetic conditions are frequent in intensively ill children. Intensive Care Med. 45, 627-636. doi: 10.1007/s00134-019-05552-x

Gubbels, C. S., VanNoy, G. E., Madden, J. A., Copenheaver, D., Yang, S., Wojcik, M. H., et al. (2020). Prospective, phenotype-driven selection of critically ill neonates for rapid exome sequencing is associated with high diagnostic yield. Genet. Med. 22, 736-744. doi: 10.1038/s41436-019-0708-6

Heringer, J., Boy, S. P., Ensenauer, R., Assmann, B., Zschocke, J., Harting, I., et al. (2010). Use of guidelines improves the neurological outcome in glutaric aciduria type I. Ann. Neurol. 68, 743-752. doi: 10.1002/ana.22095

Honzik, T., Tesarova, M., Magner, M., Mayr, J., Jesina, P., Vesela, K., et al. (2012). Neonatal onset of mitochondrial disorders in 129 patients: clinical and laboratory characteristics and a new approach to diagnosis. J. Inherit. Metab. Dis. 35, 749-759. doi: 10.1007/s10545-011-9440-3
Husami, A., Slone, J., Brown, J., Bromwell, M., Valencia, C. A., and Huang, T. (2020). Clinical utility of whole genome sequencing for the detection of mitochondrial genome mutations. J. Genet. Genomics 47, 167-169. doi: 10 . 1016/j.jgg.2020.03.001

Imai-Okazaki, A., Kishita, Y., Kohda, M., Mizuno, Y., Fushimi, T., Matsunaga, A., et al. (2019). Cardiomyopathy in children with mitochondrial disease: prognosis and genetic background. Int. J. Cardiol. 279, 115-121. doi: 10.1016/j.ijcard. 2019.01.017

Ismail, I. T., Showalter, M. R., and Fiehn, O. (2019). Inborn Errors of Metabolism in the Era of Untargeted Metabolomics and Lipidomics. Metabolites 9:242. doi: 10.3390/metabo9100242

Kingsmore, S. F., Cakici, J. A., Clark, M. M., Gaughran, M., Feddock, M., Batalov, S., et al. (2019). A Randomized, Controlled Trial of the Analytic and Diagnostic Performance of Singleton and Trio, Rapid Genome and Exome Sequencing in Ill Infants. Am. J. Hum. Genet. 105, 719-733. doi: 10.1016/j.ajhg.2019.08.009

Kishnani, P. S., Austin, S. L., Abdenur, J. E., Arn, P., Bali, D. S., Boney, A., et al. (2014). Diagnosis and management of glycogen storage disease type I: a practice guideline of the American College of Medical Genetics and Genomics. Genet. Med. 16:e1. doi: 10.1038/gim.2014.128

Kisler, J. E., Whittaker, R. G., and McFarland, R. (2010). Mitochondrial diseases in childhood: a clinical approach to investigation and management. Dev. Med. Child Neurol. 52, 422-433. doi: 10.1111/j.1469-8749.2009.03605.x

Morava, E., van den Heuvel, L., Hol, F., de Vries, M. C., Hogeveen, M., Rodenburg, R. J., et al. (2006). Mitochondrial disease criteria: diagnostic applications in children. Neurology 67, 1823-1826. doi: 10.1212/01.wnl.0000244435.27645.54

Petrikin, J. E., Cakici, J. A., Clark, M. M., Willig, L. K., Sweeney, N. M., Farrow, E. G., et al. (2018). The NSIGHT1-randomized controlled trial: rapid wholegenome sequencing for accelerated etiologic diagnosis in critically ill infants. NPJ Genom. Med. 3:6. doi: 10.1038/s41525-018-0045-8

Petrikin, J. E., Willig, L. K., Smith, L. D., and Kingsmore, S. F. (2015). Rapid whole genome sequencing and precision neonatology. Semin. Perinatol. 39, 623-631. doi: 10.1053/j.semperi.2015.09.009

Pronicka, E., Piekutowska-Abramczuk, D., Ciara, E., Trubicka, J., Rokicki, D., Karkucinska-Wieckowska, A., et al. (2016). New perspective in diagnostics of mitochondrial disorders: two years' experience with whole-exome sequencing at a national paediatric centre. J. Transl. Med. 14:174. doi: 10.1186/s12967-0160930-9

Puertas, M. J., and Gonzalez-Sanchez, M. (2020). Insertions of mitochondrial DNA into the nucleus-effects and role in cell evolution. Genome 63, 365-374. doi: 10.1139/gen-2019-0151

Puusepp, S., Reinson, K., Pajusalu, S., Murumets, U., Oiglane-Shlik, E., Rein, R., et al. (2018). Effectiveness of whole exome sequencing in unsolved patients with a clinical suspicion of a mitochondrial disorder in Estonia. Mol. Genet. Metab. Rep. 15, 80-89. doi: 10.1016/j.ymgmr.2018.03.004

Ramos, A., Santos, C., Mateiu, L., Gonzalez Mdel, M., Alvarez, L., Azevedo, L., et al. (2013). Frequency and pattern of heteroplasmy in the complete human mitochondrial genome. PLoS One 8:e74636. doi: 10.1371/journal.pone.0074636

Richards, S., Aziz, N., Bale, S., Bick, D., Das, S., Gastier-Foster, J., et al. (2015). Standards and guidelines for the interpretation of sequence variants: a joint consensus recommendation of the American College of Medical Genetics and Genomics and the Association for Molecular Pathology. Genet. Med. 17, 405-424. doi: 10.1038/gim.2015.30

Riley, L. G., Cowley, M. J., Gayevskiy, V., Minoche, A. E., Puttick, C., Thorburn, D. R., et al. (2020). The diagnostic utility of genome sequencing in a pediatric cohort with suspected mitochondrial disease. Genet. Med. 22, 1254-1261. doi: 10.1038/s41436-020-0793-6

Sanford, E. F., Clark, M. M., Farnaes, L., Williams, M. R., Perry, J. C., Ingulli, E. G., et al. (2019). Rapid Whole Genome Sequencing Has Clinical Utility in Children in the PICU. Pediatr. Crit. Care Med. 20, 1007-1020. doi: 10.1097/ PCC.0000000000002056

Saunders, C. J., Miller, N. A., Soden, S. E., Dinwiddie, D. L., Noll, A., Alnadi, N. A., et al. (2012). Rapid whole-genome sequencing for genetic disease diagnosis in neonatal intensive care units. Sci. Transl. Med. 4:154ra135. doi: 10.1126/ scitranslmed.3004041

Schaefer, A. M., Taylor, R. W., Turnbull, D. M., and Chinnery, P. F. (2004). The epidemiology of mitochondrial disorders-past, present and future. Biochim Biophys Acta 1659, 115-120. doi: 10.1016/j.bbabio.2004.09.005 
Schon, K. R., Ratnaike, T., van den Ameele, J., Horvath, R., and Chinnery, P. F. (2020). Mitochondrial Diseases: a Diagnostic Revolution. Trends Genet. 36, 702-717. doi: 10.1016/j.tig.2020.06.009

Skladal, D., Halliday, J., and Thorburn, D. R. (2003). Minimum birth prevalence of mitochondrial respiratory chain disorders in children. Brain 126, 1905-1912. doi: 10.1093/brain/awg170

Smigiel, R., Biela, M., Szmyd, K., Bloch, M., Szmida, E., Skiba, P., et al. (2020). Rapid Whole-Exome Sequencing as a Diagnostic Tool in a Neonatal/Pediatric Intensive Care Unit. J. Clin. Med. 9:2220. doi: 10.3390/jcm9072220

Stevenson, D. A., and Carey, J. C. (2004). Contribution of malformations and genetic disorders to mortality in a children's hospital. Am. J. Med. Genet. A 126A, 393-397. doi: 10.1002/ajmg.a.20409

Tulinius, M. and Oldfors, A. (2011). Neonatal muscular manifestations in mitochondrial disorders. Semin. Fetal Neonatal. Med. 16, 229-235. doi: 10.1016/ j.siny.2011.04.001

van Diemen, C. C., Kerstjens-Frederikse, W. S., Bergman, K. A., de Koning, T. J., Sikkema-Raddatz, B., van der Velde, J. K., et al. (2017). Rapid Targeted Genomics in Critically Ill Newborns. Pediatrics 140:e20162854. doi: 10.1542/ peds.2016-2854

Wang, H., Qian, Y., Lu, Y., Qin, Q., Lu, G., Cheng, G., et al. (2020). Clinical utility of 24-h rapid trio-exome sequencing for critically ill infants. NPJ Genom. Med. 5:20. doi: 10.1038/s41525-020-0129-0

Willig, L. K., Petrikin, J. E., Smith, L. D., Saunders, C. J., Thiffault, I., Miller, N. A., et al. (2015). Whole-genome sequencing for identification of Mendelian disorders in critically ill infants: a retrospective analysis of diagnostic and clinical findings. Lancet Respir. Med. 3, 377-387. doi: 10.1016/S2213-2600(15) 00139-3

Witters, P., Saada, A., Honzik, T., Tesarova, M., Kleinle, S., Horvath, R., et al. (2018). Revisiting mitochondrial diagnostic criteria in the new era of genomics. Genet. Med. 20, 444-451. doi: 10.1038/gim.2017.125

Wortmann, S. B., Koolen, D. A., Smeitink, J. A., van den Heuvel, L., and Rodenburg, R. J. (2015). Whole exome sequencing of suspected mitochondrial patients in clinical practice. J. Inherit. Metab. Dis. 38, 437-443. doi: 10.1007/ s10545-015-9823-y
Wortmann, S. B., Mayr, J. A., Nuoffer, J. M., Prokisch, H., and Sperl, W. (2017). A Guideline for the Diagnosis of Pediatric Mitochondrial Disease: the Value of Muscle and Skin Biopsies in the Genetics Era. Neuropediatrics 48, 309-314. doi: 10.1055/s-0037-16 03776

Yang, H., Yin, F., Gan, S., Pan, Z., Xiao, T., Kessi, M., et al. (2020). The Study of Genetic Susceptibility and Mitochondrial Dysfunction in Mesial Temporal Lobe Epilepsy. Mol. Neurobiol. 57, 3920-3930. doi: 10.1007/s12035-02001993-4

Yu, H., Zhang, V. W., Stray-Pedersen, A., Hanson, I. C., Forbes, L. R., de la Morena, M. T., et al. (2016). Rapid molecular diagnostics of severe primary immunodeficiency determined by using targeted next-generation sequencing. J. Allergy Clin. Immunol. 138, 1142-1151. doi: 10.1016/j.jaci.2016. 05.035

Conflict of Interest: JW, CW, and VZ were employed by AmCare Genomics Lab.

The remaining authors declare that the research was conducted in the absence of any commercial or financial relationships that could be construed as a potential conflict of interest.

Publisher's Note: All claims expressed in this article are solely those of the authors and do not necessarily represent those of their affiliated organizations, or those of the publisher, the editors and the reviewers. Any product that may be evaluated in this article, or claim that may be made by its manufacturer, is not guaranteed or endorsed by the publisher.

Copyright (c) 2021 Ouyang, Zhang, Zhang, Luo, Zhang, Hu, Liu, Zhong, Zeng, Xu, Bai, Wong, Wang, Wang, Wang and Zhang. This is an open-access article distributed under the terms of the Creative Commons Attribution License (CC BY). The use, distribution or reproduction in other forums is permitted, provided the original author(s) and the copyright owner(s) are credited and that the original publication in this journal is cited, in accordance with accepted academic practice. No use, distribution or reproduction is permitted which does not comply with these terms. 